\title{
La producción de sigillatae hispánicas mediante cocción de tipo indirecto y four à tubulures en la península ibérica. Síntesis, observaciones técnicas y propuesta tecnológica para su interpretación
}

\author{
Hispanic terra sigillata production by indirect-flame and four à \\ tubulures in the Iberian peninsula. Synthesis, technical \\ observations and technological proposal for its interpretation
}

\author{
Pablo Ruiz Montes ${ }^{1}$ \\ Universidad de Granada
}

\begin{abstract}
RESUMEN
Los procedimientos para la cochura de barnices rojos sinterizados en los grandes focos productores de sigillatae en Hispania, así como las estructuras que su puesta en práctica conlleva, aún hoy día, y a pesar del conocimiento y modelos generados en el ámbito académico, especialmente francés, han permanecido en gran medida fuera del núcleo de la investigación sobre los procesos productivos de la cerámica de época romana en la península ibérica. Con el acercamiento que ahora realizamos se pone de relieve, a partir del análisis crítico de ciertas estructuras de cocción y cerámicas arqueológicas procedentes de centros productores como Los Villares de Andújar-Isturgi, la existencia de más de un modelo para la obtención de sigillatae de un alto estándar cualitativo y barnices rojos sinterizados. A la vez, se define la implantación geográfica de los hornos denominados de conductos perimetrales en el marco de un evidente fenómeno de transferencia tecnológica con origen en las provincias galas al norte de los Pirineos y circunscrito a la mitad septentrional de la península en torno, a grandes rasgos, al foco productor de Tricio y el valle del Ebro.
\end{abstract}

\section{SUMMARY}

The procedures of firing sintered red slip potteries in the major sigillatae production centers in Hispania, as well as the structures that their implementation entails, even today, and despite the knowledge generated in the French academia, have largely remained outside the core of the research on the productive processes of Roman pottery wares in the Iberian peninsula. With the approach that we are now carrying out, it

${ }^{1}$ prmontes@ugr.es / ORCID iD: https://orcid.org/00000002-3595-4184 is highlighted, from the critical analysis of certain firing structures and archaeological ceramics coming from centers such as Los Villares de Andújar-Isturgi, the existence of not only a single model to obtain Hispanic terra sigillatae of a high qualitative standard and totally sintered red slips on it. At the same time, the geographical implantation of so-called perimeter duct kilns is defined within the framework of an evident of technological transfer process originating in the central Gaulish provinces, circumscribed to the northern half of Hispania.

PALABRAS CLAVE: cerámica romana altoimperial; barnices rojos sinterizados; hornos de conductos perimetrales; procesos de producción; eficiencia económica; Los Villares de Andújar-Isturgi; Tritium Magallum; Hispania.

KEY WORDS: Roman pottery; sintered red slip; perimeter ducts kilns; production processes; economic efficiency; Los Villares de Andújar-Isturgi; Tritium Magallum; Hispania.

CÓMO CITAR ESTE ARTÍCULO / CITATION: Ruiz Montes, P. 2020: "La producción de sigillatae hispánicas mediante cocción de tipo indirecto y four à tubulures en la península ibérica. Síntesis, observaciones técnicas y propuesta tecnológica para su interpretación", Archivo Español de Arqueología 93, 219-228. https://doi.org/10.3989/ aespa.093.020.011

En la historiografía tradicional el discurso sobre la producción de sigillatae con barnices sinterizados y acabados de calidad equiparable a los estándares de la denominada sigilleé vraie (Picon 2002: 151), ha estado indefectiblemente unido al empleo de técnicas de cochura de tipo indirecto, es decir, en estructuras de cocción y hornos modificados mediante el concurso 
de elementos intermedios, en especial los four à tubulures (p. ej. Vernhet 1981; Picon 1998), con el objeto de hacer cualitativa y cuantitativamente más eficiente el proceso de producción de estos vasos. Sin embargo, en las grandes áreas productoras de estos barnices rojos en la península ibérica, especialmente en Tritium e Isturgi, las evidencias sobre el empleo de esta técnica son, por el momento, muy puntuales.

El objetivo fundamental será ahora el de, a la luz de algunos recientes datos y hallazgos relativos a estructuras de cocción para la elaboración de sigillatae de calidad en la zona septentrional de la península, o respecto a un mejor conocimiento técnico de las series de barniz rojo sinterizado hispánico originarias de Los Villares de Andújar, introducir nuevos elementos con los que propiciar un debate riguroso acerca de, en esencia, la idoneidad de extrapolar a la península ibérica determinados modelos elaborados a partir del caso francés y de los talleres gálicos.

A la vez, es nuestra intención realizar nuevas propuestas e hipótesis de carácter tecnológico acerca de este tipo de hornos bicamerales de conductos perimetrales, así como del proceso de cocción adoptado por los maestros de los talleres productores de sigillatae clásicas en el Mediterráneo occidental.

\section{LOS FOUR À TUBULURES PERIMETRALES Y SU IMPLANTACIÓN GEOGRÁFICA EN LA PENÍNSULA IBÉRICA}

Como decimos, si hacemos caso a la historiografía dominante, para la producción de cerámicas sigillatae con revestimientos impermeables sinterizados ${ }^{2}$, se debía recurrir a la cocción indirecta por calor de radiación mediante tubi insertos en las perforaciones por toda la superficie de la parrilla de los hornos, según el modelo tradicional a imagen de las reconstrucciones efectuadas del gran horno de La Graufesenque (p. ej. Picon y Vernhet 2008). Los conductos, en contacto directo con los gases y el aire caliente, se calentaban para desempeñar de este modo su papel como emisores de calor, a la vez que dirigían hacia el exterior humos que podían resultar nocivos para el acabado exquisito que requerían vajillas de cerámica fina como la sigillata. En la base de esta clase de hornos se encuentra el proceso de cocción definido como de tipo C (Picon 1973) en el que todas las fases del mismo

\footnotetext{
${ }^{2}$ No son de consideración en nuestro trabajo las producciones con revestimientos semisinterizados no vitrificados propias, por ejemplo, de talleres menores cuyos productos son objeto de una comercialización a corta distancia; véase el caso de las sigillatae hispánicas de los alfares de Cartuja (Granada).
}

vienen caracterizadas por una atmósfera oxidada de principio a fin de la cochura.

Sin embargo, este modelo y la cocción de tipo indirecto se han materializado de manera más frecuente en estructuras u hornos de planta circular llamados de tubi o conductos perimetrales, en los que tubos o, incluso, imbrices se disponen adosados a las paredes del laboratorio para conducir el aire caliente. Este tipo de hornos son relativamente comunes y bien conocidos desde inicios de los años 90 del pasado siglo, sobre todo, en el centro y este de Francia (Dufaÿ 1996: 304305) -e incluso Inglaterra, en Colchester (Hull 1963: 20-34) y Alemania, p. ej., en Rheinzabern (Reutti 1984; Reutti y Schulz 2010)- pero también en el sur, en Montans (Schaad 2007a); muy importante, no siempre empleados para la cocción de sigillatae, como en Luxeuil-les-Bains (Card 2008) (Fig. 1) y con dataciones que oscilarán entre mediados del siglo I d. C. para el de Montans, y el segundo tercio del IV en el caso del de Mareuil-lès-Meaux (Bet et alii 2003) (Fig. 2). Destaca, por poner un ejemplo, el horno 54 de la officina de Vieux-Fresne (Saone-et-Loire), el cual presenta 20 conductos perimetrales y dos toberas perforadas en una parrilla de $0,80 \mathrm{~m}$, sostenida mediante muros paralelos atravesados por un corredor central (Notet 1996: 51-53) (Fig. 3); la planta de la cámara de combustión responde a la del tipo cuadrangular II/b de Cuomo di Caprio (1971-1972), si bien la de la parte aérea o laboratorio es circular. Este hecho, el de las morfologías diferenciales de cámara de combustión y laboratorio en un mismo horno, debía ser bastante más

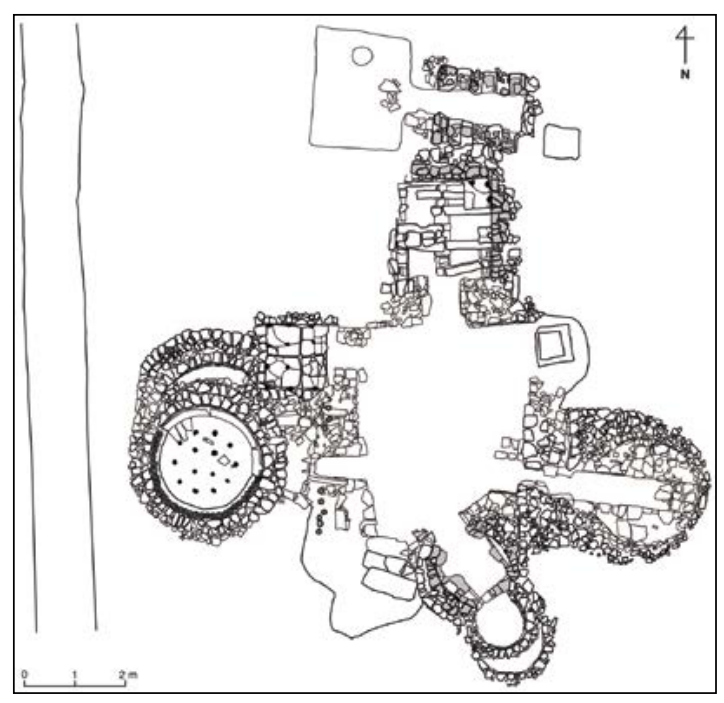

Figura 1. Planta de los hornos de Luxeuil-les-Bains. A la izquierda, es posible apreciar el horno de conductos perimetrales (según Card 2008). 


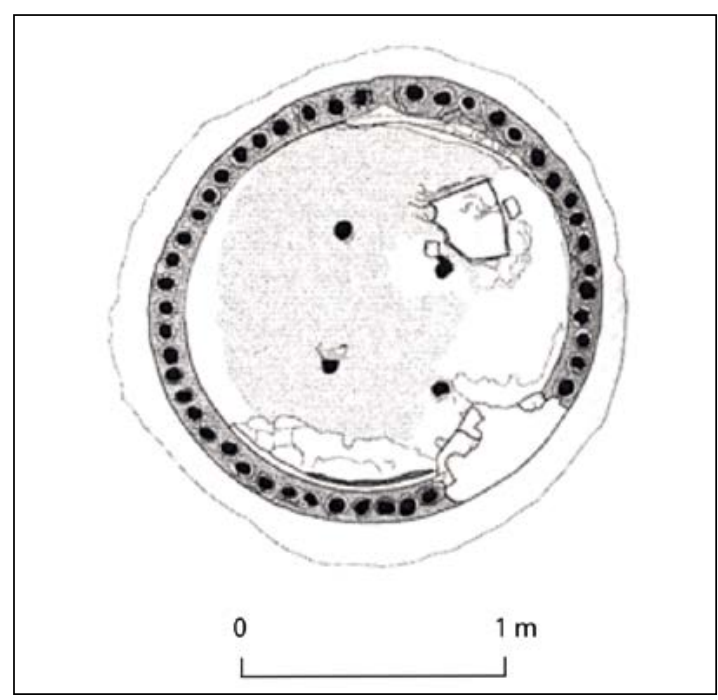

Figura 2. Detalle del horno de conductos perimetrales de Mareuil-lès-Meaux (según Bet et alii 2003).

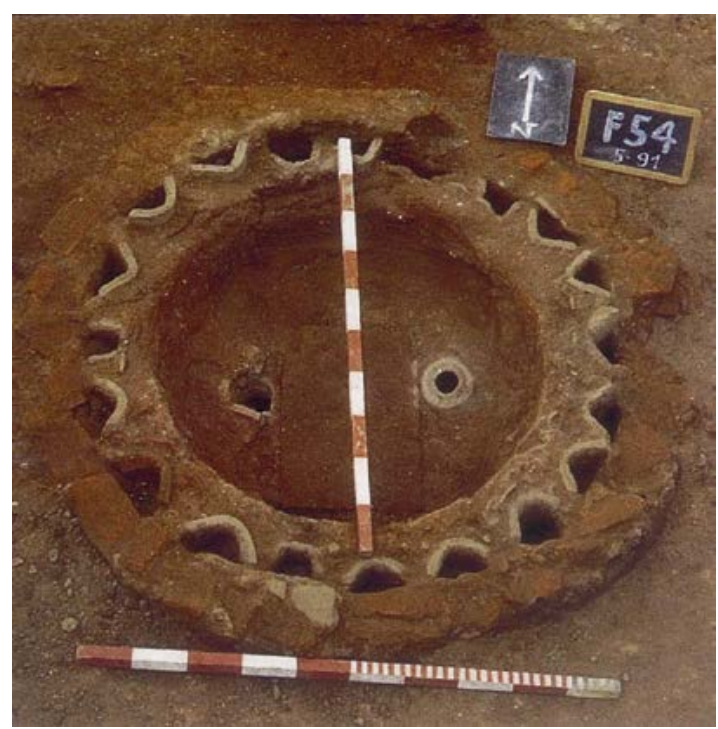

Figura 3. Vista de la pequeña piroestructura de conductos perimetrales de Vieux-Fresne (Saone-et-Loire) (según Notet 1996: 51-53).

frecuente de lo que pensamos y se encuentra, quizá, en la base de la nueva reinterpretación del gran horno de La Graufesenque (Schaad 2007b, 2007c) (Fig. 4). No obstante, la solución de muretes paralelos y corredor central en cámaras de combustión de planta circular Cuomo I/d, como en Rheinzbern, parece haber sido uno de los recursos habituales.

En la península ibérica, a pesar de la importante producción de terra sigillata de calidad y barnices

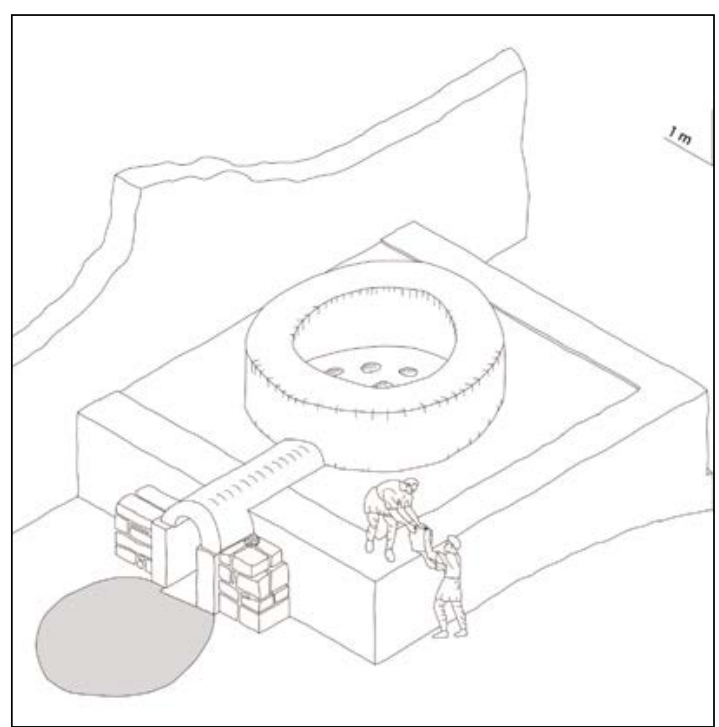

Figura 4. Reconstrucción y vista axonométrica de la propuesta de $\mathrm{Ph}$. Schaad para el gran horno de La Grausenque (según Schaad 2007c).

totalmente sinterizados -sobre todo en toda la región en torno a Tritium Magallum, en la mitad norte, y en el suburbium de Isturgi, para la mitad sur- los hornos de este tipo son muy escasos por el momento y se concentran en la mitad septentrional; los dos ejemplos que responden más claramente a esta morfología dedicados a la producción de sigillatae son los del alfar de Villarroya de la Sierra (Medrano y Díaz 2000: 274-275) y el recientemente descubierto en La Salceda, en Tricio (Gil y Luezas 2015). Este último se encuentra parcialmente excavado en el terreno y casi por completo realizado en adobes de tamaño regular. La parrilla, con un diámetro de $2 \mathrm{~m}$, estuvo perforada por cuatro toberas centrales, mientras que a lo largo de todo su perímetro se disponen los tubi perimetrales que adquieren, en este caso, la forma de piezas cerámicas semicilíndricas. Este sistema de conductos perimetrales parece haber sido revestido de barro a modo de sellado, siendo algo más grueso en las uniones entre ellos. Se trata, obviamente, de un horno bicameral de planta circular cuya infraestructura aparece caracterizada por una cámara de combustión organizada en muros radiales para determinar una planta alveolar asimilable al tipo 3B de Coll (p. ej. 2005) o I/b de Cuomo (Fig. 5).

También los dos hornos de Villarroya de la Sierra, muy parecidos al anterior, deben responder a esta tipología de horno. Aunque no tenemos muchos datos sobre la morfología exacta de la cámara de combustión, la cámara de cocción del Horno 1 (Fig. 6), que es el mejor conservado, dispone igualmente de un siste- 

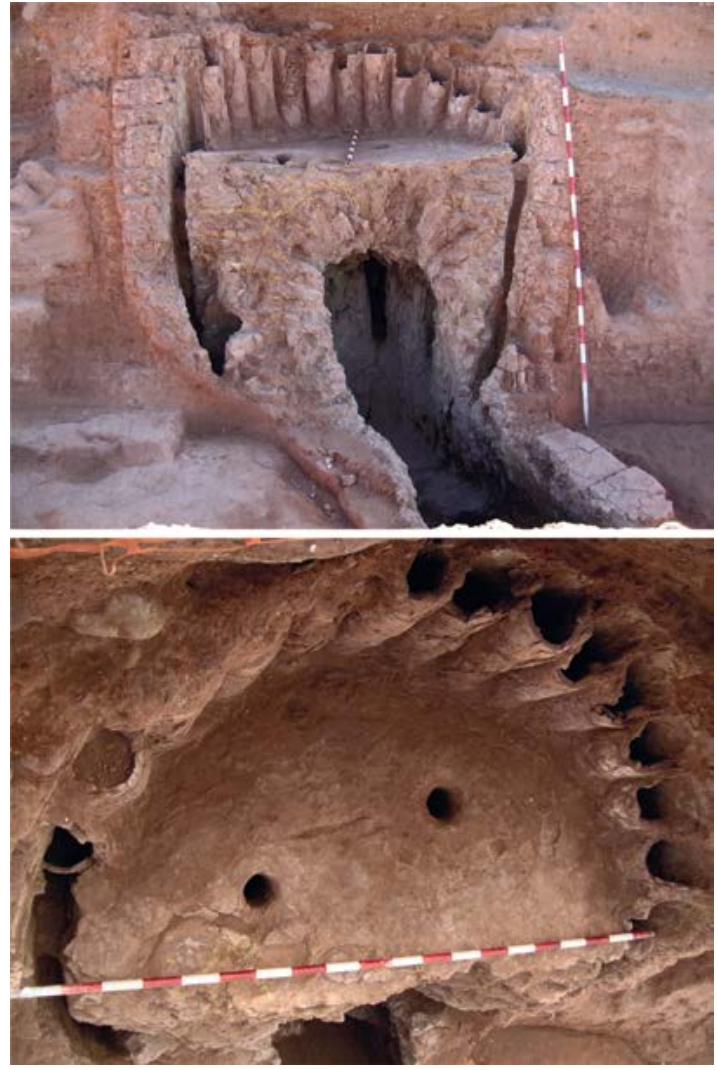

Figura 5. Dos vistas del horno de tubi perimetrales de La Salceda (Tricio) (según Gil y Luezas 2015).

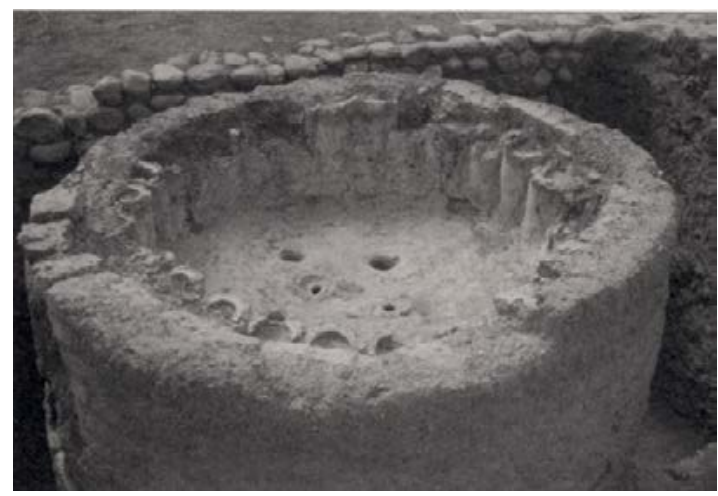

Figura 6. Imagen del laboratorio del horno de conductos perimetrales de Villarroya de la Sierra (Zaragoza) (según Medrano y Díaz 2000).

ma de toberas perimetrales -hasta 24 tubi-y una parrilla de 2,12 m de diámetro interno perforada por otras 4 toberas. El taller, según sus excavadores y a partir del repertorio producido, permaneció activo desde fines del siglo I hasta el IV d. C. (Medrano y
Díaz 2000: 277), sin especificar datación concreta alguna para las piroestructuras.

De adscripción posible, aunque menos clara dado el estado de conservación deficiente, podría ser el horno número 1 del grupo de los excavados en El Quemao, esta vez sí, datando su amortización a fines del siglo I o comienzos del siglo II d. C., y cuya planta y dimensiones son prácticamente idénticas a los vistos anteriormente (Sáenz 2000: 296-297).

A pesar de tratarse del principal foco productor de cerámicas de barniz rojo sinterizado de la Hispania meridional durante el Alto Imperio, de los seis hornos hasta el momento conocidos en Isturgi, ninguno responde a este tipo de horno de tubos perimetrales como veremos a continuación.

\section{EL CASO DE LOS VILLARES DE ANDÚJAR- ISTURGI}

La morfología general de los hornos de Los Villares de Andújar se puede resumir en pocos puntos: son de planta circular y doble cámara, la superior o cámara de cocción y la inferior o cámara de combustión a la que se accedería a través de un corredor, el praefurnio. Ambas cámaras están separadas por un piso perforado o parrilla sostenido por un pilar central. La cámara inferior o de combustión se presenta excavada en el terreno, y al que se adosa un muro perimetral compuesto de adobes. En esta cámara inferior un pilar central rectangular de ángulos redondeados en la parte posterior y achaflanados en la anterior, ayuda a sustentar una parrilla perforada, que en su parte inferior presenta una falsa bóveda resultado de la inclinación de los muros de la cámara de combustión hacia el centro (Ruiz 2011a, 2014).

De ese modo, estas estructuras de cochura aprovechaban el tiro y la circulación del aire y los gases calientes por convección. La cocción de las piezas, por tanto, se realizaba por el contacto del aire caliente con ellas; dicho tiro, a su vez, permitía ser regulado de diversas maneras: obturación de las perforaciones, disposición estratégica de la carga en el laboratorio, disposición del hogar en la cámara inferior, etc. Directamente asociado a este tipo de tecnología de cocción se encuentra el proceso de cochura de los vasos definido por Picon (1973) como tipo A, en el que se alterna fases de cocción esencialmente reductora a causa de la acción de los gases y humos de la combustión en el laboratorio, con un enfriamiento predominantemente en atmósfera oxidante en la que los humos dejan paso al oxígeno que ahora circula libremente a través de la cámara de cocción. Las cerámicas, que anteriormente habrán sufrido una reducción del óxido férrico 
en su composición -lo que les proporcionará una tonalidad gris-, al inicio de la fase de enfriamiento, cuando aún las temperaturas son altas, experimentarán una reoxidación a través de la restitución del óxido férrico y la formación de nuevos cristales de hematites. Entonces, las cerámicas vuelven a adquirir tonalidades claras con coloraciones distintas atendiendo a su composición química, entre el rojo pálido, pasando por naranjas claros hasta beige y amarillos. Como venimos observando en las características físicas y mineralógicas de las cerámicas arqueológicas, este es el proceso de cocción seguido por los maestros horneros de Los Villares de Andújar para la cochura de las cerámicas pertenecientes al grupo químico LVA 1 (Ruiz 2014), aunque ya sabemos que también lo fueron las cerámicas comunes béticas y las cerámicas de cocina producidas en estos mismos talleres (Peinado 2010), y presumiblemente los moldes en cerámica para la elaboración de sigillatae decoradas, a tenor de las coloraciones de sus pastas, claramente encuadrables junto a las vinculadas al primero de los grupos químicos de Andújar que acabamos de mencionar.

Por su parte, si tenemos en cuenta los parámetros técnicos determinados para el grupo de referencia LVA 2 de sigillatae hispánicas de Los Villares de Andújar-Isturgi (Fig. 7), datadas en la fase inicial de la producción de barnices rojos en torno a mediados del siglo I d. C. (Ruiz 2011b: 172-219; Ruiz 2014), deberíamos aceptar, a priori, que en estos talleres el proceso de cocción de tipo $\mathrm{C}$ debió ser el procedimiento habitual. Especialmente llamativa es la TCE estimada para estas, nunca por encima de los 950/1000 ${ }^{\circ} \mathrm{C}$; son aquellas que ofrecen a simple vista los mejores acabados y calidades técnicas de todas las sigillatae locales producidas a lo largo de la vida de los talleres isturgitanos. De la microscopía electrónica de barrido (en adelante, MEB) se desprendía la alta calidad del barniz empleado en estas piezas, en un estadio de vitrificación total, adherente y con un grosor uniforme de unos $20 \mu \mathrm{m}$ en todas las partes de la pieza. Este acabado de la superficie en un vaso cocido a una temperatura relativamente baja con respecto a lo que por muchos investigadores se considera terra sigillata de buena factura producida siempre por encima del rango mencionado, únicamente es posible siguiendo un estricto y experimentado proceso de selección y acondicionamiento de los sedimentos arcillosos empleados en la fabricación de la pasta y el barniz, lo que permitirá, obteniendo el tamaño de grano más apropiado, aún a estas temperaturas no excesivamente elevadas alcanzar un importante grado de sinterización ${ }^{3}$ en los recubrimientos de estas piezas. Todo esto demuestra, en definitiva, la posibilidad real de obtener terra sigillata de un alto nivel técnico y con barnices con un importante, cuando no completo grado de impermeabilización sin la necesidad de alcanzar temperaturas en el rango de los $1050-1100{ }^{\circ} \mathrm{C}$ que en principio, según lo generalmente aceptado, no sería posible desarrollar con garantías de éxito sin el concurso de los llamados hornos de radiación o cualquier otro sistema de llama indirecta (four à tubulures), en contraposición al tipo de horno de tiro vertical directo.

Sin embargo, ninguna de las estructuras conocidas allí responden a la tipología de hornos de conductos perimetrales que debería llevar aparejada esta técnica de cochura y varios elementos de consideración nos plantean muy serias dudas al respecto. En primer lugar, y como se ha podido observar en aquellos hornos que conservaban la parrilla intacta, la localización más bien desordenada y poco lógica de las aberturas por las que circularía el aire caliente y donde se dispondrían insertas las columnas de tubi, no ayudaría en ningún caso al proceso de carga y a la disposición

\footnotetext{
${ }^{3}$ Recordemos que este es el término empleado para designar el proceso de densificación y vitrificación que al final lleva al revestimiento arcilloso de la sigillata a su estado de porosidad mínima (Cuomo di Caprio 2007: 91, 314-317, 2017: 235-239).
}
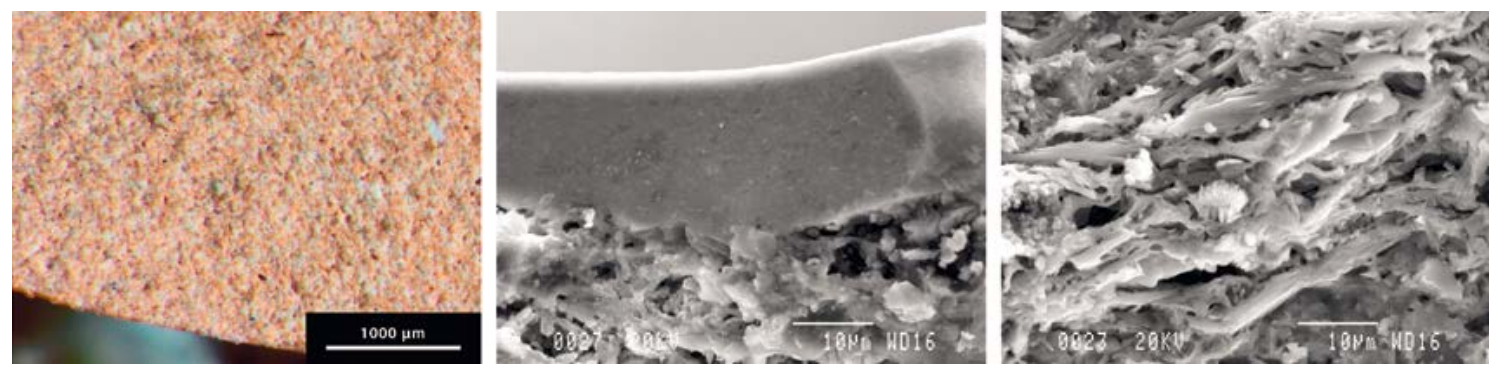

Figura 7. De izquierda a derecha: fotografía con lupa binocular, MEB del revestimiento (vitrificación total) y MEB de la matriz (vitrificación inicial/continuada) de un individuo del grupo de referencia LVA 2 de Los Villares de Andújar (según Ruiz Montes 2011; 2014). 
ordenada de los vasos en el laboratorio que imprimiese, además, una mayor homogeneidad a la cocción. En segundo lugar, y no menos importante, resulta alarmante la inexistencia en el registro arqueológico vinculado a la actividad alfarera de los talleres, de trazas, artefactos o elementos cerámicos estructurales claramente asociados a su uso como conductores de calor $\mathrm{y}$, por tanto, como partes integrantes de un procedimiento técnico de implementación puramente alóctona. Y no hay que olvidar aquellos indicios claros que traíamos a primer plano, los cuales hablaban de ciertas cerámicas sigillatae desechadas a causa de su exposición a fenómenos episódicos de carburación durante la fase de enfriamiento de la cocción (Fig. 8), que no hubiesen sido posibles de ninguna manera en hornos de cocción a llama indirecta o por radiación si hacemos caso, estrictamente, del modelo establecido.

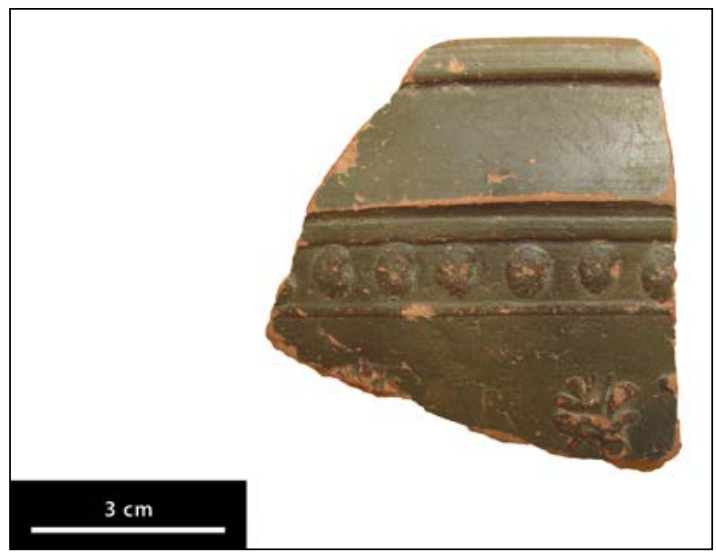

Figura 8. Fragmento de un vaso de la forma 37 de sigillata hispánica de Los Villares de Anújar cuya superficie muestra los efectos de una carburación accidental del barniz durante las últimas fases de la cocción (fotografía autor).

\section{LA DISFUNCIÓN DEL MODELO}

La disfunción se hace evidente y nos conduce a la formulación de preguntas que inciden sobre los propios fundamentos del modelo que sustenta la imagen generada y generalizada en gran parte de la historiografía moderna relativa al procedimiento técnico empleado para la obtención de las cerámicas sigillatae sinterizadas. Esta deficiencia encuentra origen, por un lado, en la excesiva dependencia del modelo ideado por los investigadores franceses y sus fours à tubuleres $\mathrm{y}$, por otro, en la extrema simplificación y escasa flexibilidad presupuesta a las técnicas de cocción y a los artesanos encargados de su realización. Si aceptamos para el maestro hornero una profunda especialización y unos conspicuos conocimientos acerca del comportamiento de una hornada, ¿por qué no hacer lo mismo con la más que probable búsqueda y experimentación por parte de estos de soluciones técnicas intermedias? Otros modelos que parecen más ajustados (Cuomo di Caprio 2007: 541; 2017: 250 ss., especialmente 339) han insinuado la posibilidad de producir verdaderas sigillatae parcialmente sinterizadas y de calidad aceptables sin el concurso específico de la cocción de tipo indirecto a través de elementos radiantes y, por consiguiente, eliminando el exagerado determinismo desempeñado por los distintos tipos de cochura en la configuración y articulación del modelo.

Según lo observado en Los Villares de Andújar y en la serie de cerámicas terra sigillata de las fases tempranas de la producción, podemos aún ir más lejos y afirmar que, al contrario de lo pensado hasta ahora, los artesanos horneros que llegaron con la tecnología de la sigillata a Isturgi se encontraban en situación de cocer barnices rojos sinterizadas con un, como diría Picon (2002), vernis grésés que impermeabiliza y le da mayor resistencia mecánica al manufacto, sirviéndose de hornos bicamerales de tiro vertical y llama directa sin la necesidad de desarrollar temperaturas elevadas de $1050-1100{ }^{\circ} \mathrm{C}$; no en vano, poco más allá de estas se sitúa el umbral que sostiene el delicado equilibrio físico de la cocción de las cerámicas antes de producirse el desastroso fenómeno de la fusión. Seguramente conscientes de estos riesgos sobre la hornada y la imposibilidad de alcanzar esas temperaturas de forma controlada en base a sus conocimientos empíricos, en Andújar nunca pretendieron alcanzar rangos tan altos.

Creemos, por tanto, que todas las sigillatae de las officinae isturgitanas debieron ser cocidas adoptando un proceso de cocción que, sin el concurso de elementos radiantes con los que intermediar en el laboratorio la difusión del calor, garantizase el predominio de una atmósfera oxidante durante las fases de cochura y enfriamiento de la hornada a través del control preciso y disciplinado de un flujo continuo de aire por la cámara de cocción. Este bien podría haberse tratado de la cocción semioxidante que autores como Montagu (1989) ya han planteado y que no supondría ningún tipo de cambio estructural en la morfología de los hornos más que la desaparición de la cubierta del laboratorio o, sencillamente, la disposición de chimeneas en ella. De esta manera, si bien el alfarero no consigue una atmósfera del todo oxidante, ni tampoco una carburación importante de las cerámicas, sí obtendrá un resultado muy similar al de la cocción del modo C. Como es lógico, todo esto supone un conocimiento profundo y especializado, además de una 
vigilancia -no solo mediante las evidencias visibles que presenta el horno, sino también gracias al empleo de muestras (¿probinas?)- y cuidados extremos por parte de los artesanos que trascendía las pautas y rudimentos puestos en práctica de manera mecánica en las cotidianas cochuras alternantes del modo A (reductor-oxidante) ya conocidas por los alfareros locales.

Últimamente, el mejor conocimiento de los hornos con conductos, así como las dificultades para identificar esos elementos estructurales (tubi) ligados a hornos alfareros fuera de las regiones mencionadas con anterioridad, generan hoy día no pocas dudas en los foros especializados referentes a algunos de los extremos asentados y generalmente aceptados por los planteamientos técnicos sobre la tecnología de fabricación de las cerámicas antiguas ideados por Verhnet y Picon, y su automática extrapolación a otros talleres en distintas zonas del Imperio.

\section{MATIZANDO EL MODELO. PROPUESTA TECNOLÓGICA}

A falta de contrastar experimentalmente, las propuestas que podamos plantear no dejan de ser hipótesis de trabajo o un punto de partida desde el cual validar los diversos puntos de que se compone. Teniendo esto presente, creemos que el modelo estructural manejado hasta el momento para el horno de conductos periféricos -y ni que decir tiene, para el gran horno de La Graufesenque en su propuesta de reconstrucción tradicional- se presta a diversas matizaciones, en su mayoría de tipo técnico, que pueden resumirse en los siguientes puntos:

- Esta variante del horno bicameral es un desarrollo técnico particular que adquiere forma en determinadas regiones del centro y este de la actual Francia, para luego extenderse hasta zonas limítrofes y alcanzando, en consecuencia, el norte de la península ibérica o el sur de Inglaterra. En cualquier caso, consideramos que esta evolución tiene lugar con posterioridad a la introducción de la técnica de elaboración de la sigillata en la Bética y en Los Villares de Andújar. Recordemos que, referido a los barnices rojos aretinos e itálicos en general, esta es una problemática totalmente abierta y lo único cierto es que hoy día no es posible asegurar con total certeza que hornos de cocción indirecta mediante elementos radiantes fuesen empleados para su cochura.

- No se trata de una morfología estructural privativa de la producción de barnices rojos sinterizados, detectándose este tipo de hornos en talleres en los que no parecen haberse elaborado estas vajillas cerámicas.
De esta manera, su empleo para la producción de clases cerámicas de menor valor añadido introduce un nuevo elemento de juicio con valor interpretativo a la hora de modular las propuestas tradicionales.

- Lo anterior hace de los hornos de conductos perimetrales una herramienta enfocada a la búsqueda de una mayor eficiencia técnica de la propia estructura, más que un horno ideado expresamente para la producción de barnices rojos sinterizados (sigilleé vraie) y las especiales condiciones de hornada que su producción requeriría. El caso de Los Villares de Andújar y la serie de sigillatae hispánicas sinterizadas de primera época (grupo de referencia LVA 2) demuestra, además, que es posible obtenerlas sin el concurso de elementos radiantes y en ambiente predominantemente oxidante o semioxidante, como diría Montagu. En este sentido, el modo $\mathrm{C}$ de cocción de Picon, a nuestro juicio se prestaría a algunas matizaciones que, sin duda, lo deben enriquecer.

- En realidad, este tipo de piroestructuras en las que el laboratorio presenta siempre una cámara de cocción de planta circular, debieron funcionar de un modo parecido al de un horno de mufla (Fig. 9). En nuestra opinión tanto las toberas perforadas en parrilla -que suelen ser menos en este tipo de hornoscomo los tubos perimetrales actuaban de manera combinada como conductores del aire caliente y generadores de ciertas corrientes convectivas en el interior del laboratorio antes de alcanzar el sistema temporal de cubierta y salir al exterior por la chimenea; por ello, consideramos que estos conductos nunca condujeron ese aire de manera directa hacia el exterior, extremo, reconocemos, difícil de contrastar arqueológicamente por la inexistencia de laboratorios que conserven la totalidad de sus paredes. De haber sido así -evacuando directamente al exterior, como normalmente se ha planteado- el derroche de combustible sería inasumible para alcanzar las temperaturas necesarias en el proceso; pero es que, además y no menos importante, tal desperdicio entraría en confrontación directa con la lógica de la eficiencia calórica que rige en este tipo de tecnologías.

- Igualmente, a mayor complejidad de la cámara superior, se intuyen ciertas soluciones estructurales en la cámara de combustión para conducir más ordenadamente los flujos de calor hacia las zonas de mayor interés. Tanto la solución de muros radiales como la de muretes paralelos atravesados por un corredor central parecen funcionar bajo ese razonamiento de mejor y más controlado reparto del calor hasta los puntos de ascenso del calor hacia el laboratorio, en especial hacia los conductos perimetrales. Ese paso se produce, o bien de manera directa, o bien mediante un canal continuo 


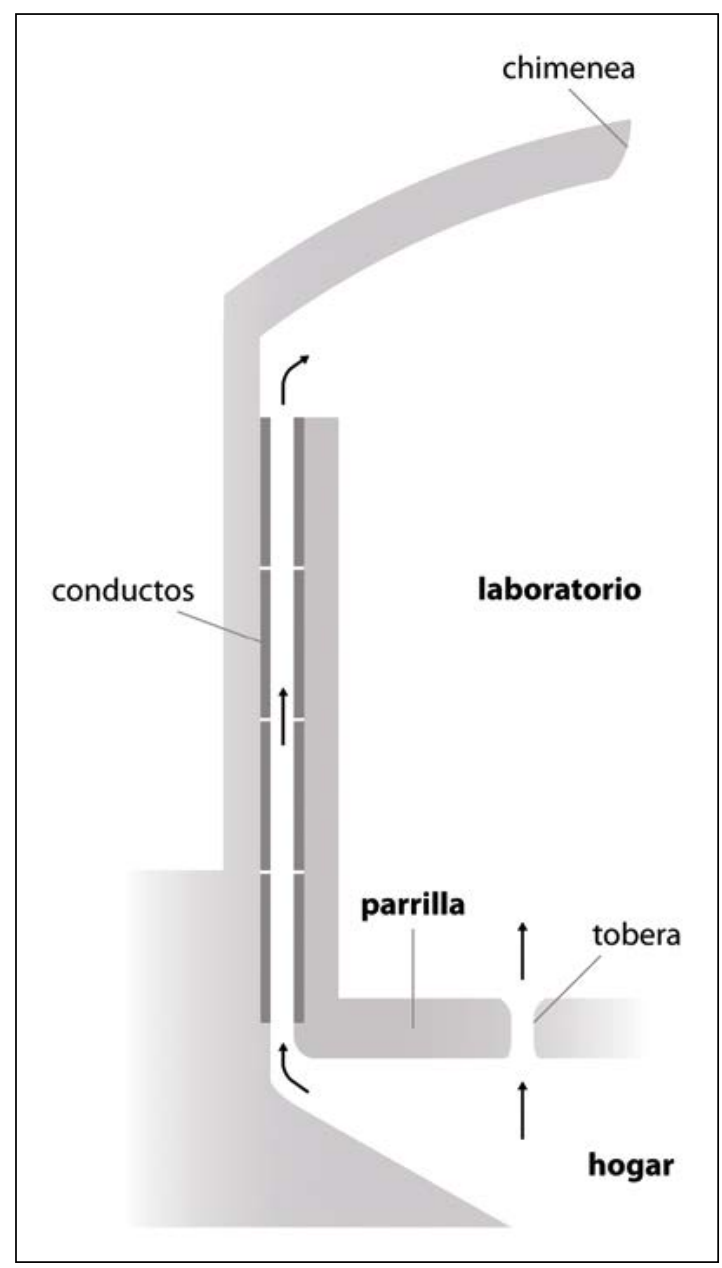

Figura 9. Esquema de la propuesta de funcionamiento térmico para los hornos de conductos perimetrales (elaboración propia).

que recorre todo el perímetro del horno a la altura de la parrilla y en el que se insertarán, a su vez, los tubi.

- La circulación perimetral de aire caliente producirá, por tanto, diversos beneficios de carácter térmico: añade estanqueidad a la estructura puesto que genera una pantalla o cortina de aire favoreciendo el aislamiento de la cámara superior lo que, a su vez, limita la incidencia negativa de la curva isoterma en su interior para, en definitiva, resultar una cocción más homogénea por la mejor distribución del calor y sin tantos golpes de fuego. Se reproducen, por decirlo de alguna manera, los efectos de una concameratio o doble pared, puede que especialmente útil, por ejemplo, en ambientes con condiciones climáticas menos cálidas.

- Podemos aceptar que la introducción de este refinamiento técnico cualitativo -el de los hornos bi- camerales de conductos perimetrales- supuso algunos ligeros cambios en el modo de proceder particular por parte del maestro hornero durante la marcha de la cochura en sus fases de acumulación de calor, mantenimiento y enfriamiento. No hay que descartar, quizá de una manera excesivamente especulativa por nuestra parte, que comportase también una menor complejidad de uso, relajando el control de una estructura que se prestará a un comportamiento menos imprevisible y más equilibrado.

\section{CONCLUSIONES}

La difusión de los hornos à tubulures en la península ibérica se presenta, a día de hoy, como un fenómeno de escasa implantación reducido a áreas limítrofes del sur de la Galia, casi exclusivamente el valle del Ebro, lo cual resulta del todo lógico por tratarse de una de las zonas más favorablemente expuestas a la recepción de contingentes de artesanos galos.

Además, la evidencia arqueológica y los resultados proporcionados por la caracterización arqueométrica de cerámicas sigillatae de calidad de otros talleres peninsulares como los de Isturgi, ponen de relieve la existencia de procedimientos alternativos al tradicionalmente planteado por el modelo francés para la elaboración de barnices rojos con revestimientos sinterizados en estado avanzado o total de vitrificación sin la necesidad del empleo de este tipo de piroestructuras, como ya viene evidenciando el caso itálico.

Pero es que, a nuestro juicio, el propio modelo francés, tanto en lo relativo a la interpretación de estas estructuras de combustión como a los procedimientos de cocción en ellos llevados a cabo, necesita de un ajuste y revisión que permita valorar en su justa medida el fenómeno de la aparición de los hornos de tubos perimetrales -no exclusivos de la producción de sigillatae-, la mecánica de funcionamiento -que debe entenderse más en la línea de lo propuesto por nosotros mismos más arriba- y, sobre todo, la determinación de las necesidades técnicas y económicas que subyacen a su surgimiento e implantación: muy alta demanda, poca disponibilidad relativa de tiempo en la preparación de las materias primas, necesidad de desarrollar elevadas temperaturas por encima de la media y minimización de fallas. Ya hemos expuesto nuestro parecer al respecto. Es decir, el four à tubulures debe entenderse como el resultado natural de la búsqueda por parte de los artesanos ceramistas de un medio con que hacer frente a una alta exigencia técnica para alcanzar una reducción importante del porcentaje de fallos o, mejor dicho, un mejor rendimiento en favor de una maximización del proceso productivo en 
el marco de aplicación de un criterio que hoy denominaríamos de "eficiencia económica".

\section{BIBLIOGRAFÍA}

Bet, Ph., Delage, R. y Van Ossel, P. 2003: "Un atelier de sigillée de type argonnais près de Meaux; le site de la Grange du Mont à Mareuil-lès-Meaux (Seine-et-Marne)", SFECAG, Actes du Congrès de Saint-Romain-en-Gal, 435-448.

Card, Ch. 2008: "Nouvelles donnés sur les productions des ateliers de potiers gallo-romains de Luxeuil-lesBains (Haute-Saône): la vaisselle en céramique non sigillée", Revue archéologique de l'Est 57, 205-226.

Coll Conesa, J. 2005: "Hornos y producción de cerámica romana en la Comunidad Valenciana”, J. Coll (coord), Recientes investigaciones sobre producción cerámica en Hispania, 155-173.

Cuomo di Caprio, N. 1971-1972: "Proposta di classificazione delle fornaci per ceramica e laterici nell'area italiana. Dalla preistoria a tutta l'epoca romana", Sibrium 11, 371-461.

Cuomo di Caprio, N. 2007: Ceramica in archeologia 2. Antiche tecniche di lavorazione e moderni metodi d'indagine, Roma.

Cuomo di Caprio, N. 2017: Ceramics in archaeology. From prehistoric to medieval times in Europe and the Mediterranean. ancient craftsmanship and modern laboratory techniques, L'Erma di Bretschneider, Roma.

Dufaÿ, B. 1996: "Les fours de potiers gallo-romains: synthèse et classification. Un nouveau panorama", SFECAG, Actes du congrès de Dijon, 297-311.

Gil Zubillaga, L. y Luezas Pascual, R. A. 2015: "El horno romano de La Salceda (Tricio, La Rioja) en

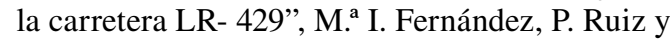
M. ${ }^{a}$ V. Peinado (eds.): Terra Sigillata Hispánica, 50 años de investigaciones, Roma, 137-149.

Hull, M. R. 1963: The Roman potters' kilns of Colchester, Reports of the research committee of the Society of Antiquaries of London 21, Oxford.

Medrano Marqués, M. y Díaz Sanz, M. A. 2000: "El alfar romano, villa y necrópolis de Villarroya de la Sierra (Zaragoza)", Salduie 1, 273-282.

Montagu, J. 1989: Les secrets de fabrication des céramiques antiques, Saint-Vallier-sur-Rhône.

Notet, J. C. 1996: "Ultimes recherches sur l'officine cèramique du Vieux-Fresnes á Gueugnon (Saoneet-Loire): Présentation de quelques résultats remarquables", SFECAG, Actes du Congrés du Dijon, 51-62.

Peinado Espinosa, M. ${ }^{\text {a }}$ V. 2010: Cerámicas comunes romanas en el Alto Guadalquivir. El alfar de Los
Villares de Andújar, Tesis doctoral, Universidad de Granada.

Picon, M. 1973: Introduction à l'étude technique des céramiques sigillées de Lezoux, Centre de Recherches sur les techniques Gréco-romaines 2, Dijon.

Picon, M. 1998: "Les tubulures et les supports d'étagères du gran four à sigillées de La Graufesenque, et le céramiques calcaires de l'Antiquité", Annales de Pegasus 3, 32-40.

Picon, M. 2002: "Les mode de cuisson, les pâtes et les vernis de La Graufesenque: una mise au point", G. Genin y A. Vernhet (dirs.), Céramiques de la Graufesenque et autres productions d'époque romaine. Nouvelles recherches, Milleu, 139-164.

Picon, M. y Vernhet, A. 2008: "Les très grands fours à sigillées en Gaule, et notamment à la Graufesenque: Observations techniques", SFECAG. Actes du Congrès de L'Escala-Empuries, 553-566.

Reutti, F. 1984: Neue archäologische Forschungen im römischen Rheinzabern, Karlsruhe.

Reutti, F. y Schulz, R. 2010: "Brennöfen für Terra Sigillata in Rheinzabern. Befunde und Rekonstruktion", RCRF Acta 41, 567-587.

Ruiz Montes, P. 2011a: "Romanización, producción de cerámicas y ¿transferencias tecnológicas? en el Alto Guadalquivir. A propósito de los hornos romanos de Los Villares de Andújar", J. Abellán, C. Lazarich y V. Castañeda (coords.), Homenaje al profesor Antonio Caro Bellido, vol. II, Cádiz, 339-362.

Ruiz Montes, P. 2011b: Nuevas lecturas en torno a procesos de producción y sustratos productivos en el complejo alfarero romano de Los Villares de Andújar (Andújar, Jaén), Tesis doctoral, Universidad de Granada, Granada.

Ruiz Montes, P. 2014: Romanización y producción de cerámicas finas en las áreas periféricas de la provincia Bética. Factores de implantación, comercio y desarrollo técnico en el suburbium artesanal de Isturgi Triumphale (Los Villares de Andújar, Jaén) (ss. I-II d.n.e), BAR International Series 2642, Oxford.

Sáenz, M. ${ }^{a}$ P. 2000: "Avance sobre la excavación del centro alfarero romano de 'El Quemao' (Tricio, La Rioja)", Salduie 1, 295-302.

Schaad, D. 2007a: "Les fours à tubulures", D. Schaad (dir.): La Graufesenque (Millau, Aveyron), I, Condatomagos, une agglomération de confluence en territoire rutène (II a.C-II d.C.), Santander, 219225.

Schaad, D. 2007b: “Le 'grand four' de La Graufesenque et un four à sigillées de Montans: étude comparative", Aquitania XXIII, 171-183. 
Schaad, D. 2007c: "Le grand four", D. Schaad (dir.), La Graufesenque (Millau, Aveyron), I, Condatomagos, une agglomération de confluence en territoire rutène (II a.C-II d.C.), Santander, 190-206.
Vernhet, A. 1981: "Un four de la Graufesenque (Aveyron): la cuison des vases sigillés", Gallia 39-1, $25-43$.

Recibido: 05-04-2019

Aceptado: 07-02-2020 